\title{
SOME ACCURATE SOLUTIONS OF THE LIFTING SURFACE INTEGRAL EQUATION
}

\author{
E. O. TUCK ${ }^{1}$
}

(Received 18 December 1991; revised 31 July 1992)

\begin{abstract}
This note describes a simple numerical method for solution of the lifting surface integral equation of aerodynamics, and provides benchmark computations of up to 7 figure accuracy for flat rectangular wings of arbitrary aspect ratio. The nature of the large aspect ratio limit is also investigated numerically and asymptotically. This enables determination of the limiting behaviour near the wing tips, which is compared to the predictions of lifting line theory. Generalisations to non-rectangular wings are discussed.
\end{abstract}

\section{Introduction}

The lifting surface equation is

$$
\iint_{B} \gamma(\xi, \eta) W(x-\xi, y-\eta) d \xi d \eta=-4 \pi U f_{x}(x, y)
$$

where

$$
W(X, Y)=Y^{-2}(1+X / R)
$$

with $R=\sqrt{X^{2}+Y^{2}}$. This equation is derived in standard aerodynamic texts, e.g., Ashley and Landahl ([1], page 132), Thwaites ([10], page 301).

Equation (1.1) is an integral equation over a finite region $B$ of the $(x, y)$ plane, namely the projection on that plane of the planform of a thin wing. The unknown $\gamma(x, y)$ of the integral equation is the "bound vorticity", which is equal to the jump in the streamwise component of velocity across the wing, and hence

\footnotetext{
'Applied Mathematics Department, The University of Adelaide, S.A. 5005, Australia

(C) Australian Mathematical Society, 1993, Serial-fee code 0334-2700/93
} 
is proportional to the jump in pressure across the wing. The quantity $\gamma(x, y)$ is therefore an output of prime aerodynamic interest, and for example the net lift is proportional to its integral over $B$, the lift coefficient being

$$
C_{L}=-\frac{2}{U B} \iint_{B} \gamma(x, y) d x d y
$$

where we have also used the symbol $B$ for the actual area of the planform.

The right-hand side of (1.1) is in general a given function of $x$ and $y$ on $B$, proportional to the forward speed $U$ of the wing and to the local angle of attack $-f_{x}(x, y)$ of the mean surface $z=f(x, y)$ of the wing. In this paper we shall generally assume a flat plate at uniform angle of attack $\alpha$; thus $f_{x}=-\alpha$, and the whole right-hand side of (1.1) is a constant over all of $B$.

The kernel $W(X, Y)$ of the integral equation is a unit horseshoe vortex. It is the value on $z=0$ of a singular solution of the three-dimensional Laplace equation. There is a strong (formally non-integrable) singularity at $Y=0$ (i.e., at $\eta=y$ ) for all $X>0$, i.e., $x>\xi$, which represents physically the vortex sheet trailing aft of the wing. Formally we need a Hadamard interpretation of the divergent integral, but this is by-passable whenever we can write down a formal anti-derivative.

And indeed we can, several times, noting that

$$
W=K_{X X Y}
$$

where

$$
K(X, Y)=X \log (Y+R)+(1 / 2) Y \log (X+R)-X Y^{-1}(X+R) / 2
$$

with

$$
\begin{gathered}
K_{X}=\log (Y+R)-Y^{-1}(X+R)+1 \\
K_{X X}=-X^{-1} Y^{-1}(X+R)+X^{-1}
\end{gathered}
$$

and

$$
K_{X Y}=Y^{-2}(X+R) \text {. }
$$

There is no unique solution to (1.1), and its general solution depends on one arbitrary function of $y$. To render the solution unique, we must apply a subsidiary condition at each value of $y$. This requires us to specify the boundary curve of $B$ in several segments, including leading edges, tips, and trailing edges. Although there are various complicated possibilities for non-convex planforms, we shall here assume a simple convex $B$ as in Figure 1, bounded on the left by a single leading edge curve $x=x_{L}(y), 0<y<s$ and on the right by a single 
(a) PLAN VIEW

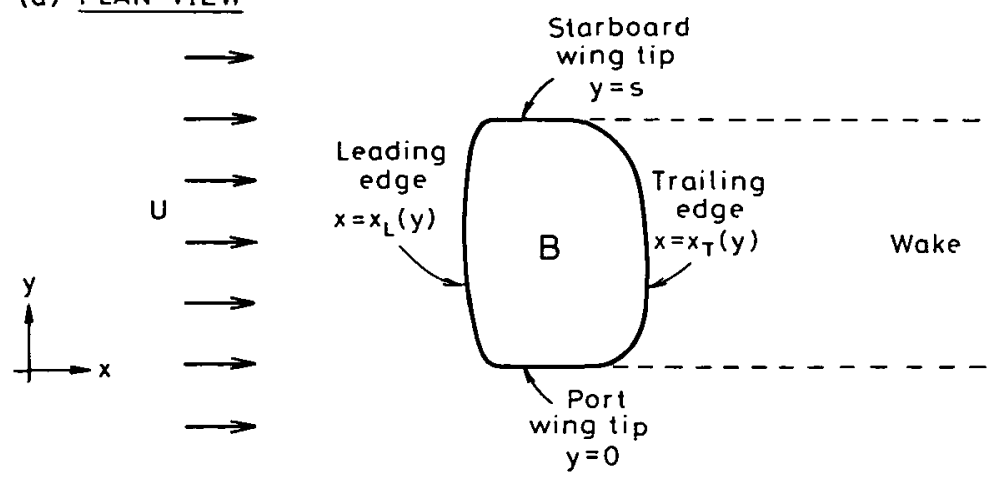

(b) SIDE VIEW

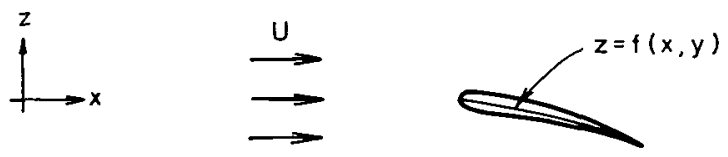

FIGURE 1. A general planform $B$.

trailing edge curve $x=x_{T}(y), 0<y<s$, where $s$ is the span of the wing. The Kutta condition then states that the bound vorticity vanishes on the trailing edge, i.e.,

$$
\gamma\left(x_{T}(y), y\right)=0, \quad 0<y<s .
$$

Note that there is no necessity to have $x_{L}=x_{T}$ at either tip $y=0$ or $y=s$. If the leading and trailing edges, uniquely defined for convex $B$, do not meet, there must be a tip portion of the boundary of $B$, where $y=0$ for $x_{L}(0)<x<x_{T}(0)$, or $y=s$ for $x_{L}(s)<x<x_{T}(s)$. These tips consist of straight ends parallel to the stream direction, and are not often present on actual wings, which (for good aerodynamic reasons) tend to have rounded ends, where the leading and trailing edges meet at a point. However, they are essentially present and of non-zero length $c$ for the idealised rectangular $c \times s$ planforms to be used as an example here, which have $x_{L}=0$ and $x_{T}=c$ (for all $y$ between 0 and $s$ ), where $c$ is the chord. In principle, such a finite-length tip segment parallel to the stream direction need be neither leading or trailing; however, we shall see that it is 
effectively a trailing edge for the rectangular wing, since (1.9) happens to be satisfied on it.

The lifting surface equation (1.1) cannot be solved analytically for arbitrary $B$. However, an explicit solution has been obtained for elliptic $B$ by Hauptmann and Miloh [5]. When $B$ is a circle, this solution for $\gamma(x, y)$ is expressed in the form of a series of associated Legendre functions, and the lift coefficient (1.3) is found to be

$$
C_{L} / \alpha=32 /\left(8+\pi^{2}\right)=1.7907503 .
$$

Otherwise, we must resort to numerical methods. The heroic attempts of the aerodynamicists to solve this equation numerically date back to well before the computer age. This history is well surveyed in Thwaites [10]. A somewhat more recent survey is given by Wang [14], and a typical recent paper is that by Frink [3]. Examples of applications where numerical solution of (1.1) is used are in Kerwin and Lee [6] and Xü [15].

The last references use what is perhaps the most successful numerical method for solution of the lifting surface equation, namely the so-called "vortex-lattice" method. In that method, the wing is represented by a collection of isolated point horseshoe vortices. In effect, the smooth bound vorticity function $\gamma(x, y)$ in (1.1) is replaced by a sum of Dirac delta functions. The location of these delta functions and the choice of collocation points for satisfaction of (1.1) is of crucial importance for the success of this method.

In the following, we describe an alternative numerical method which provides (in principle) a somewhat smoother and less sensitive numerical method, by representing the vorticity distribution by a step function rather than a delta function. Even-smoother approximations to $\gamma(x, y)$ could be also be used. However, even the vortex lattice method is capable of yielding results of high accuracy, and in particular (J.E. Kerwin, private communication) confirms the 7-figure accurate computations of the lift coefficient of the rectangular wing to be presented below, with a comparable computational effort.

\section{Numerical method}

Before discretisation, it is convenient to integrate (1.1) once with respect to $x$, obtaining

$$
\iint_{B} \gamma(\xi, \eta) K_{X Y}(x-\xi, y-\eta) d \xi d \eta=-4 \pi U f(x, y)+C(y)
$$


for some arbitrary function of integration $C(y)$. This function $C(y)$ must eventually be determined by use of the Kutta condition (1.9), but we can postpone that determination, temporarily assuming that the right-hand side of (2.1) is known. Then (2.1) is an integral equation with a kernel $K_{X Y}$ given by (1.8), and has a unique solution that is straightforward to find by elementary numerical methods.

Namely, suppose we discretise $B$ into a finite number $N$ of rectangular panels $B_{j}$. On each panel, we assume that the bound vorticity is a constant $\gamma_{j}$. This step function approximation is the only approximation that has to be made; everything else is done exactly.

Thus the left hand side of (2.1) becomes

$$
\sum_{j=1}^{N} \gamma_{j} \iint_{B_{j}} \frac{\partial^{2} K}{\partial \xi \partial \eta} d \xi d \eta
$$

and the integral in (2.2) can be evaluated explicitly for rectangular $B_{j}$, in terms of values of the kernel $K$ given by (1.5) at the four corners of $B_{j}$. If we then force (2.1) to hold at some point $\left(x_{i}, y_{i}\right) \in B_{i}$ inside the $i$-th panel, we obtain a system of $N$ linear algebraic equations in $N$ unknowns, of the form

$$
\sum_{j=1}^{N} A_{i j} \gamma_{j}=b_{i}
$$

where $b_{i}$ is the value of the right-hand side of $(2.1)$ at $\left(x_{i}, y_{i}\right)$ and

$$
\begin{aligned}
A_{i j}= & K\left(x_{i}-\xi_{j}^{++}, y_{i}-\eta_{j}^{++}\right)+K\left(x_{i}-\xi_{j}^{--}, y_{i}-\eta_{j}^{--}\right) \\
& -K\left(x_{i}-\xi_{j}^{+-}, y_{i}-\eta_{j}^{+-}\right)-K\left(x_{i}-\xi_{j}^{-+}, y_{i}-\eta_{j}^{-+}\right) .
\end{aligned}
$$

Here $\left(\xi_{j}^{++}, \eta_{j}^{++}\right)$is the top right corner of $B_{j},\left(\xi_{j}^{+-}, \eta_{j}^{+-}\right)$the bottom right corner, etc.

In principle, all we have to do is to invert the matrix $\left[A_{i j}\right]$ to solve the problem, and indeed this can be made into a satisfactory numerical method, in spite of the fact that the function $C(y)$ is still unknown, and hence the right hand side vector $b_{i}$ is (in part) unknown. Suppose there are $n<N$ such unknown values of $C(y)$. Then we must solve (2.3) $n+1$ times, with $n$ artificial right hand sides. The required final solution is a linear combination with $n$ coefficients, that are found at the last step by forcing satisfaction of the Kutta condition $n$ times. This method has been used with success on equivalent one-dimensional airfoil equations (Tuck $[11,12]$ ), where $n=1$, since there is no $y$-dependence. 


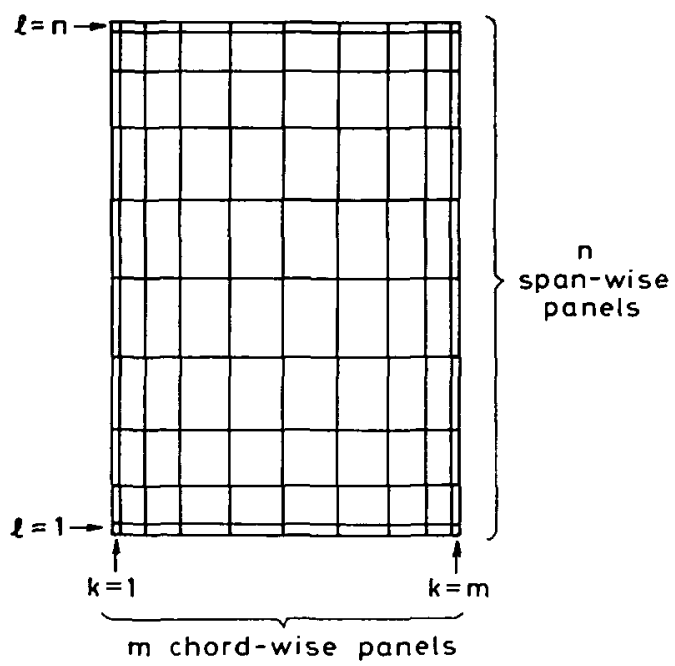

FIGURE 2. Panelisation for rectangular $B$.

A somewhat more efficient method when $n>1$, as is necessarily the case for the present two-dimensional integral equation, is to build the satisfaction of the Kutta condition into the solution process at an earlier stage. A relatively crude satisfaction of (1.9) is achieved by setting $\gamma_{j}=0$ on $n$ panels adjacent to the trailing edge. Then, having dispensed with these $n$ unknowns, we replace them by $n$ values of the unknown function $C(y)$, re-defining $\gamma_{j}=C\left(y_{j}\right)$ for those panels. This idea was used by Oertel [6] for a hydrodynamic planing problem

A slightly more accurate method of satisfaction of (1.9) builds in the expected "square root" rate at which the bound vorticity tends to zero as we approach the trailing edge, and is described later, but the resulting modification is a minor one. Essentially, the present numerical method is as described above, and can be used for any planform that can be satisfactorily discretised into rectangular panels. Since a planform that is itself a rectangle is clearly in that category, let us first describe the detail of the method for that case.

\section{Discretisation of rectangular planforms}

We divide the rectangle into $N=m \times n$ panels, as in Figure 2. That is, suppose $x=X_{k}$ for $k=0,1,2, \ldots, m$ is a discretisation of the chord length $x=c$, with $X_{0}=0, X_{m}=c$, and $y=Y_{l}$ for $l=0,1, \ldots, n$ is a discretisation 
of the span $s$, with $Y_{0}=0, Y_{n}=s$. If the pair of integers $(k, l)$ defines the panel that is the $k$-th from the leading edge and the $l$-th from the port wingtip, this must be the $j$-th panel over all, where $j=k+(l-1) m$. Thus the coordinates of the corners of the $j$-th panel, needed for evaluation of the matrix elements $A_{i j}$ by (2.4), are given by

$$
\xi_{j}^{++}=\xi_{j}^{+-}=X_{k}, \quad \xi_{j}^{--}=\xi_{j}^{-+}=X_{k-1}
$$

and

$$
\eta_{j}^{++}=\eta_{j}^{-+}=Y_{l}, \quad \eta_{j}^{--}=\eta_{j}^{+-}=Y_{l-1} .
$$

The collocation point $\left(x_{j}, y_{j}\right)$ is similarly defined in terms of values $x=\bar{X}_{k}$ of $x$ lying in the $k$-th chordwise segment, and values $y=\bar{Y}_{l}$ of $y$ lying in the $l$-th spanwise segment.

In principle, any mesh and any set of collocation points will work, including the obvious choices of uniform meshes, and of mid-points as collocation points. But Chebyshev meshes and collocation points work better. Namely, we set

$$
\begin{aligned}
X_{k} & =\frac{c}{2}[1-\cos (k \pi / m)], \\
\bar{X}_{k} & =\frac{c}{2}\left[1-\cos \left(\left(k-\frac{1}{2}\right) \pi / m\right)\right], \\
Y_{l} & =\frac{s}{2}[1-\cos (l \pi / n)], \\
\bar{Y}_{l} & =\frac{s}{2}\left[1-\cos \left(\left(l-\frac{1}{2}\right) \pi / n\right)\right] .
\end{aligned}
$$

This puts fine meshes near the borders of the planform, at exactly the rate they are needed.

Now the simplest method, as discussed in the previous section, for satisfaction of the Kutta condition is simply to set $\gamma_{j}=0$ for $j=l m, l=1,2, \ldots, n$. Then we have no further use for $A_{i j}$ when $j=l \mathrm{~m}$. So we now re-define $\gamma_{j}=C\left(\bar{Y}_{l}\right)$ for those $j$, and transfer these new unknowns to the left of (2.1). The net effect is that when $j=l m$, there are replacement matrix elements $A_{i j}=-1$ if $i=k+(l-1) m, k=1,2, \ldots, m$, (i.e. if the collocation point lies on the $l$-th chordwise strip), and $A_{i j}=0$ otherwise. The right-hand side vector $b_{i}$ is now simply

$$
b_{i}=-4 \pi U f\left(x_{i}, y_{i}\right),
$$

and the solution is completed by solution of the system (2.3), using any convenient matrix inversion package. 
This method works well enough, but can be improved by noting that when the bound vorticity tends to zero at the trailing edge, as required by the Kutta condition, it does so like the square root of distance from that edge. Hence, instead of forcing $\gamma_{j}=0$ when $j=l m$, we can extrapolate $\gamma_{j}$ from $\gamma_{j-1}$ in such a way as to model vanishing of the vorticity at the trailing edge itself, rather than (less accurately) at a collocation point inside a panel adjacent to that edge.

When we are using the Chebyshev chordwise collocation points (3.4), it is particularly easy to accomplish this, since the local effect is precisely such as to make $\gamma$ tend to zero linearly with a stretched coordinate proportional to $m-k+1 / 2$. Since this quantity takes the value $1 / 2$ at the collocation point of the $m$-th segment, and $3 / 2$ at the corresponding point of the $(m-1)$-st segment, we must have $\gamma_{j}=\gamma_{j-1} / 3$ when $j=l m$. This correction can then be implemented very easily; instead of simply discarding the original matrix element $A_{i j}$ when $j=l m$, we add one-third of its value to $A_{i, j-1}$, and then discard it, replacing it as before by either -1 or 0 .

\section{Computational experience}

An important measure of the efficiency of this sort of program is the rate at which the final answer, in this case the lift coefficient defined by (1.3) (evaluated by summation of the computed vorticity $\gamma_{j}$ times the area of its panel $B_{j}$ ), converges to a constant limiting value as the number $N$ of panels is increased. For example, a uniform grid with collocation at mid-points and the simplest Kutta procedure gives only an $O\left(N^{-1 / 2}\right)$ rate. Actually, this convergence rate is $\mathrm{O}\left(m^{-1}\right)+\mathrm{O}\left(n^{-1}\right)$, when both $m$ and $n$ are large, with $N=m n$, and it is preferable to consider the separate influences of the chordwise $(m)$ and spanwise (n) discretisations.

Moving to a Chebyshev grid (3.3) in the chordwise direction gives a considerable improvement in the rate of convergence with respect to $m$, which is now $\mathrm{O}\left(\mathrm{m}^{-2}\right)$, even if collocation still takes place at actual mid-points of segments, and this rate does not improve further if collocation takes place at Chebyshev "mid-points" (3.4). However, somewhat surprisingly, the reverse is true for spanwise discretisation. There is no improvement in the rate of convergence with respect to $n$ by moving to a Chebyshev discretisation (3.5), so long as collocation still takes place at actual mid-points of segments. However, a dramatic improvement is achieved when collocation then takes place at Chebyshev points (3.6). This improvement seems to make the convergence occur even faster than 


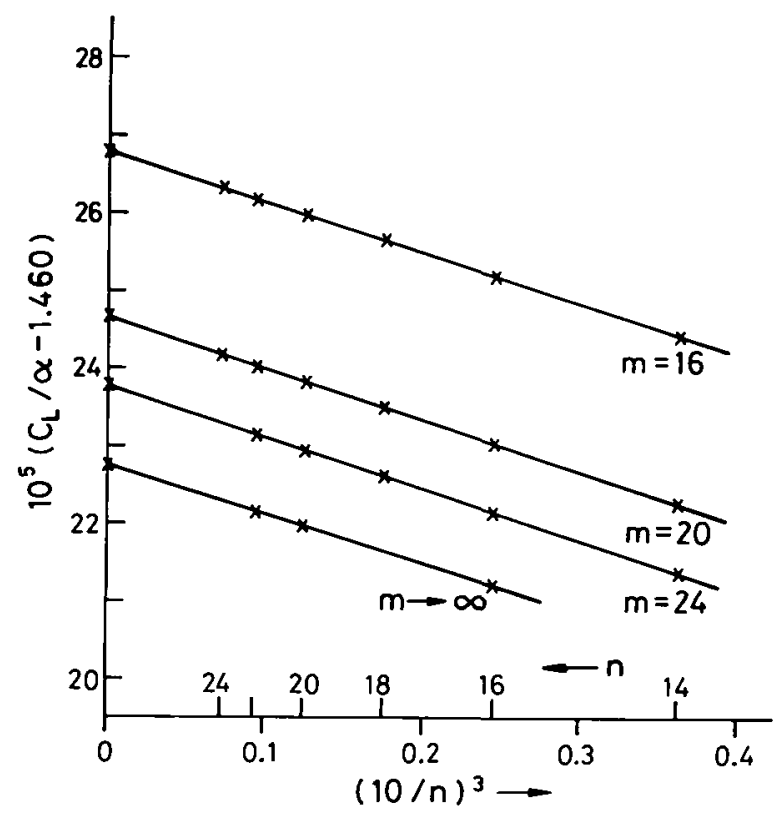

FIGURE 3. Convergence rate with number $n$ of spanwise segments.

an inverse square rate, up to a rate close to $\mathrm{O}\left(n^{-3}\right)$.

The final improvement in convergence rate is obtained by using higher accuracy satisfaction of the Kutta condition, as described above. Note that when using this technique, one must remember to include a contribution to the lift coefficient from the trailing segments, corresponding to a vorticity equal to one third of that on the next upstream segment. Obviously this change has a greater effect on the chordwise discretisation (3.3), (3.4) than the spanwise discretisation (3.5), (3.6), though it does make the latter appear to produce even closer to an $\mathrm{O}\left(n^{-3}\right)$ rate. The effect on the chordwise discretisation is to increase the rate to slightly better than $O\left(m^{-3.5}\right)$.

Figures 3 and 4 (for a flat square wing) illustrate the accuracy of the final method. The horizontal axis of Figure 3 is proportional to the inverse cube of $n$, and the results at fixed $m$ fall very close to straight lines. Similarly, the horizontal axis of Figure 4 is proportional to the inverse 3.5-th power of $m$, and again results at fixed $n$ are close to straight lines.

The final accuracy is well represented by these figures, which suggest the 


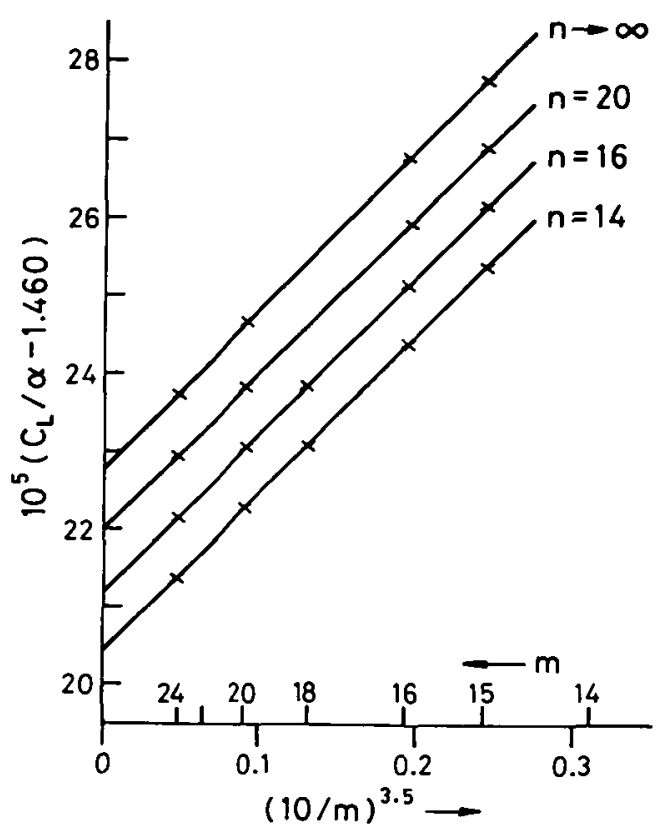

FIGURE 4. Convergence rate with number $m$ of chordwise segments.

formula

$$
C_{L} / \alpha=1.460227+0.00022(10 / m)^{3.5}-0.00007(10 / n)^{3} .
$$

We thus predict that the correct lift coefficient slope for the square wing is $C_{L} / \alpha=1.460227$, with confidence about all 7 figures ( 6 decimal places). A typical actual computation with 400 panels, namely at $(m, n)=(20,20)$, gives $C_{L} / \alpha=1.460238$. To a certain extent, the nearly 6-figure accuracy of this computed example is a fluke, since (4.1) indicates that there are compensating errors from the chordwise and spanwise discretisation. Finite $m$ over-predicts the lift, whereas finite $n$ underpredicts it, and it is always possible to adjust $m$ and $n($ e.g., $(m, n) \approx(15,10)$ or $(25,20))$ so that the lift is apparently computed exactly, while other output measures still have non-zero errors. However, at least 6 -figure ( 5 decimal place) accuracy (on every criterion) is always obtainable by extrapolation from about 400 panels.

Even a more modest 100-panel computation with $(m, n)=(10,10)$ gives the nearly 5-figure accurate result 1.460368 . Extrapolation from coarser $(10,8)$ and $(8,10)$ computations then gives the nearly 6-figure accurate estimate 1.460217 . Again, these results are perhaps unreasonably good, due to cancellation. One 


\begin{tabular}{c|c}
$s / c$ & $C_{L} / \alpha$ \\
\hline 0.5 & 0.77352 \\
1 & 1.46023 \\
2 & 2.47446 \\
4 & 3.61205 \\
8 & 4.58606 \\
10 & 4.83848 \\
15 & 5.21907 \\
20 & 5.43349
\end{tabular}

TABLE 1. Lift coefficient slope for various aspect ratios.

way to estimate the true expected error with any $(m, n)$ grid (as if cancellation had not occurred) is to add the separate extrapolation terms in (4.1), ignoring their sign. For example, with $(m, n)=(10,10)$, this suggests an inherent error of the order of about twice the actual computed error, still about 4-figure accuracy.

In practice, only half of the above panels were used, by exploiting span-wise symmetry. This cuts the matrix inversion time, though not the matrix set-up time, which tends to dominate the present program. A double precision FORTRAN version of this program determined the above $(m, n)=(20,20)$ result in about 12 seconds on a Sun SPARC IPX workstation. Only 4 of the 12 seconds were used for inversion of the $200 \times 200$ matrix. Results with $(m, n)=(10,10)$ take less than a second to compute.

There is some loss of arithmetic precision, e.g., due to the double differencing involved with setting up the matrix in (2.4). For example, comparing the same computation in single and double precision arithmetic indicates that the former can yield at best 5-figure accuracy, so double precision is mandatory (and satisfactory) if 6-7 figures are required. This extreme accuracy is not in fact usually required for the engineering applications of interest, and about 4figure results can be achieved in single precision easily, with a grid as coarse as $(10,10)$, and even without extrapolation.

Table 1 shows results of 6-figure accuracy for the lift coefficient slope $C_{L} / \alpha$ for rectangular planforms of varying span/chord (aspect) ratio $s / c$, and these results are also graphed in Figure 5. Aside from serving as a benchmark for 


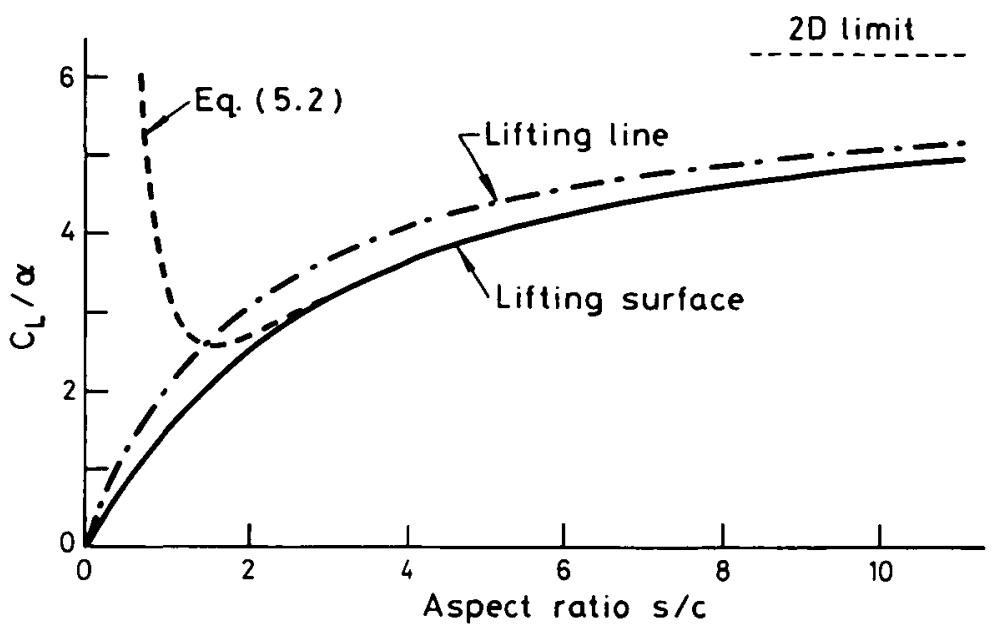

FIGURE 5. Lift coefficient slope versus aspect ratio for rectangular wings.

other solution procedures (e.g., showing that the result $C_{L} / \alpha=2.4732$ of Frink [3] at $s / c=2$ is of 3-figure accuracy), the present high-accuracy computations are useful for detailed investigation of special features, such as behaviour in the wing-tip domain, as in the following section.

\section{Large aspect ratio rectangular wings}

The lift coefficient slope tends to the two-dimensional value $2 \pi$ as the aspect ratio tends to infinity. But at what rate?

Asymptotic analysis of a lifting-line nature (Koerniawan [7]; see also Guermond [4]) suggests that

$$
C_{L} / \alpha=2 \pi-\pi \frac{c}{s}\left[\log \frac{s}{c}+q\right]+\mathrm{O}\left(\left[\frac{c}{s}\right]^{2} \log \frac{s}{c}\right)
$$

for some constant $q$. The empirical formula

$$
C_{L} / \alpha=2 \pi-\pi \frac{c}{s}\left[\log \frac{s}{c}+2.5620\right]+1.404\left[\frac{c}{s}\right]^{2}\left[\log \frac{s}{c}+3.645\right]
$$

(which also estimates the error term in (5.1)) fits our numerical results as in Table 1 to within all 6 figures of accuracy for $s / c \geq 15$ and to within 5 figure 


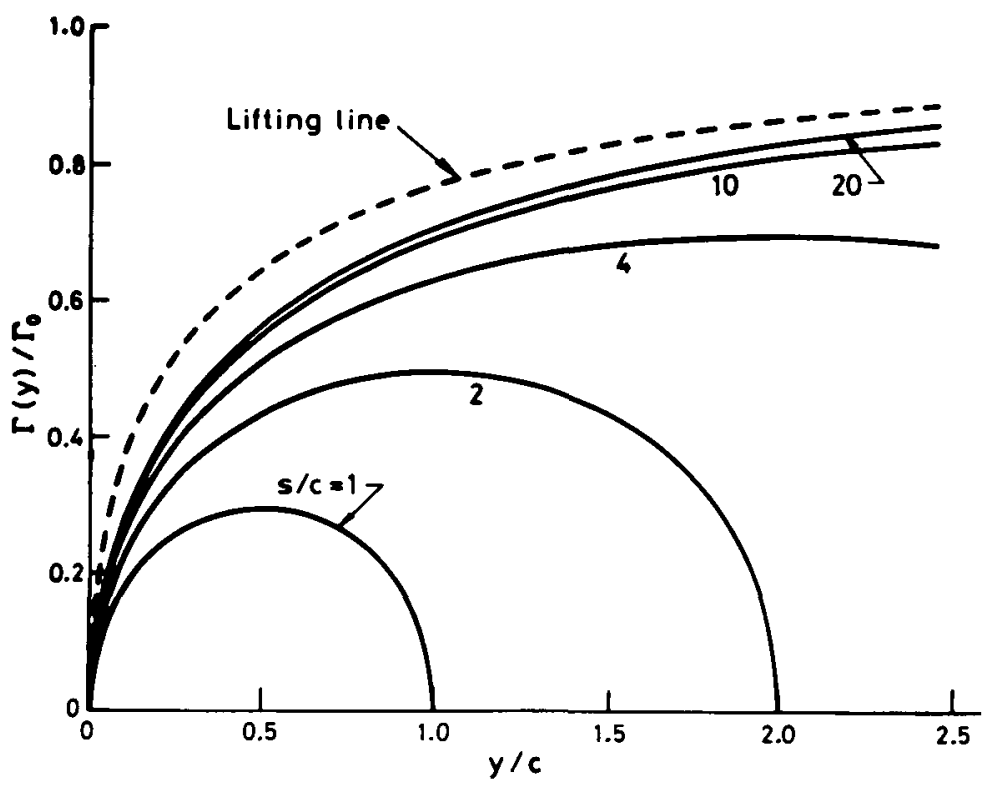

FIGURE 6. Circulation as a function of spanwise co-ordinate.

accuracy for $s / c \geq 8$. Hence we confirm (5.1), and predict $q=2.5620$. Note that it is necessary to do the original computations to nearly 7 figure accuracy in order to estimate $q$ to 5 figures, and the coefficients of the next terms in (5.2) are only known to 4 figures, even so.

Figure 5 shows (dashed) the behaviour of the asymptotic estimate (5.2) in comparison with the exact computations. The formula (5.2) retains reasonable (less than $1 \%$ error) accuracy down to aspect ratios of the order of 3 . The chaindotted curve is that given by Prandtl's lifting line equation ([1, page 142]).

The asymptotic analysis also suggests that the bound vorticity $\gamma(x, y)$ itself has an asymptotic form near the tip $y=0$, scaling with $x / c$ and $y / c$ when $y=\mathrm{O}(c)<<\mathrm{O}(s)$. For example, the total section-wise circulation

$$
\Gamma(y)=\int_{0}^{c} \gamma(x, y) d x
$$

approaches a limiting form as $s / c \rightarrow \infty$ which is a unique function of $y / c$. The range of this function is then semi-infinite, and it must approach the twodimensional value $\Gamma_{0}=-\pi U c \alpha$ for large $y / c$, i.e., far from the wing tip. Figure 6 shows $\Gamma(y) / \Gamma_{0}$, as computed by integration of our computed results, 


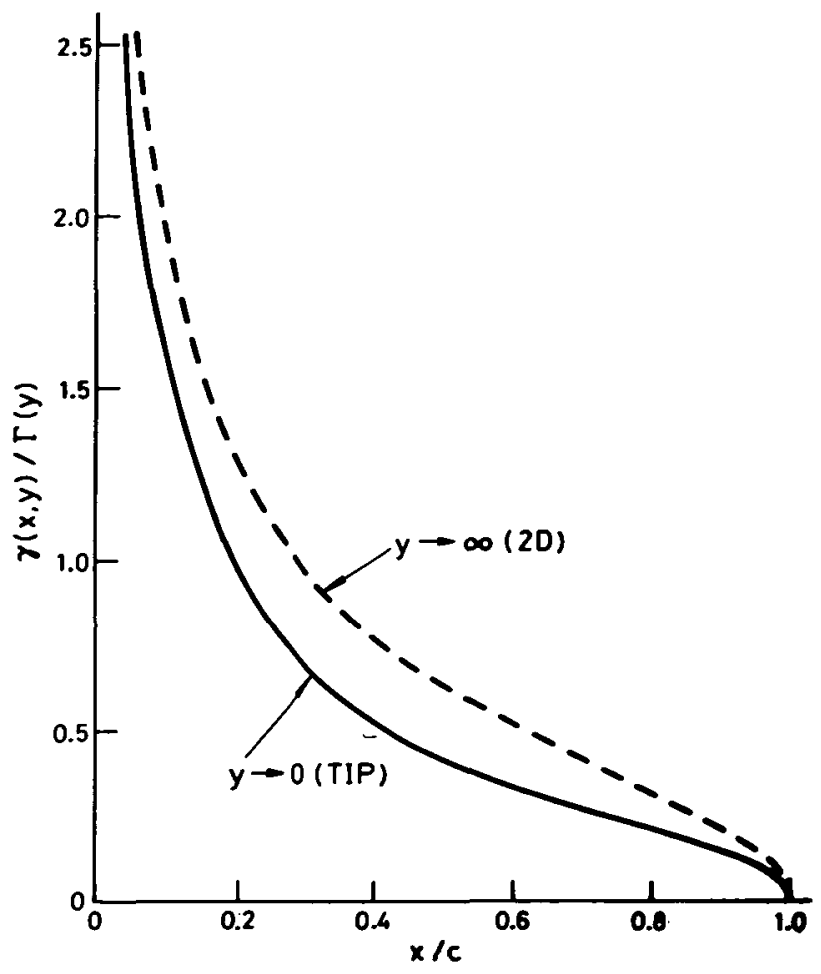

FIGURE 7. Chordwise loading distribution near wing tip.

for various aspect ratios. These results have almost become independent of aspect ratio over the range of this figure for $s / c>20$, and the curve shown for $s / c=20$ is the required limiting spanwise lift distribution near the wing tips.

Figure 6 also shows (dashed) for comparison the estimate provided by Prandtl's lifting line equation for this circulation function. Stewartson [9] was able to solve the semi-infinite-range lifting line equation exactly in terms of quadratures, and Varley and Walker [13] have recently re-derived and generalised that solution. However, (see Van Dyke [2]), the lifting line theory is not a consistent large aspect ratio asymptotic expansion and cannot be expected to retain accurate validity near the wing tips.

For example, as $y \rightarrow 0$, the circulation $\Gamma$ tends to zero as the square root of the distance $y$ from the wing tip, i.e.

$$
\Gamma(y) / \Gamma_{0} \approx k_{1}(y / c)^{1 / 2} \text { as } y \rightarrow 0
$$

for some dimensionless constant $k_{1}$. Stewartson's [9] lifting line solution has $k_{1}=4 / \pi \approx 1.2732$. Our numerical results for actual rectangular wing tips 
suggest $k_{1} \approx 0.91$. Similarly, the Stewartson solution predicts a value 1.816 for the parameter $q$ in (5.1), compared to our result $q=2.562$. Figure 7 shows the actual chordwise distribution of the bound vorticity (scaled with the local circulation $\Gamma(y)$ ); lifting line theory gives no information about the chordwise distribution. The solid curve is our result for small $y$ and large aspect ratio $s / c$, whereas the dashed curve is the two-dimensional result, i.e. the limit as $y / c \rightarrow \infty$ for large $s / c$, namely

$$
\gamma(x, y) / \Gamma(y)=\frac{2}{\pi} \sqrt{\frac{c-x}{x}} .
$$

The area under both curves is unity even though the solid curve seems to lie entirely below the dashed curve. The wing tip loading has a very much bigger contribution from the leading corner $x=0, y=0$, where it appears that $\gamma \approx x^{-3 / 4} y^{1 / 2}$, but this increased leading edge singularity with respect to $x$ is not apparent until $x$ is so small that the graph is off the scale of Figure 7 .

\section{Rectangular panels for non-rectangular wings}

The simplicity and accuracy of the present numerical method depends essentially on explicit closed form integration of the kernel as in (2.2), and this demands rectangular panels, or at least panels which are right-angled polygons. If the wing planform of interest is not rectangular, there are two ways to go.

One way is to abandon rectangular panels, at least for a band of panels adjacent to the wing edges. For example, a reasonable fit to most curved edges could be achieved if the equivalent of (2.4) was available for triangular panels. But it is not. Any attempt to use non-rectangular panels implies that approximations must be used in evaluating the integral (2.2) of the kernel function over the panel. Of course, one way to do this approximate evaluation for a small triangular panel is to split it up into many even smaller rectangles, on each of which the integral is evaluated exactly. This and other options for high-accuracy panelisation are left for further work.

A second way to approach the problem is to accept the coarseness of a rectangular panelisation, and to examine how well the aerodynamics is modelled nevertheless. If, for example, we use a $(10 \times 20)$ rectangular panelisation of a circular wing, as in Figure 8, the present program solves for flow over the actual jagged-edge planform so created, with the same ease and accuracy as if 


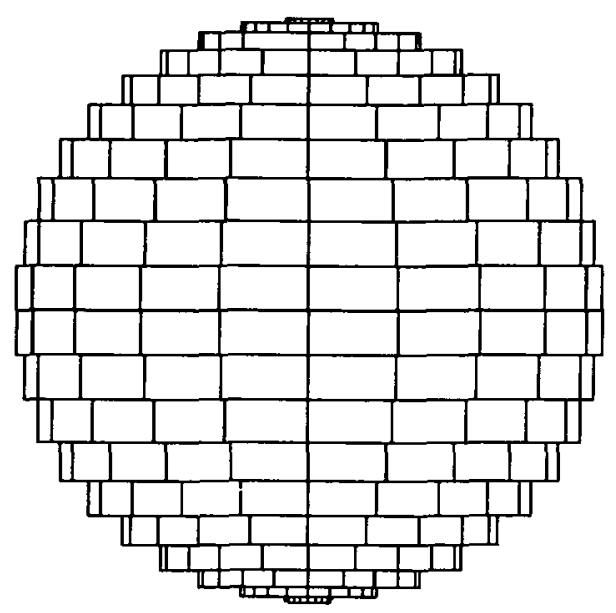

FIGURE 8. Rectangular panelisation of a circle.

the planform were rectangular. Are the results for such a jagged-edge planform close to those for a circular planform?

The only change needed for rectangular panelisation of an arbitrary convex planform is to stretch the chord-wise Chebyshev grid, namely to replace (3.3) by

$$
X_{k}=x_{L}\left(\bar{Y}_{l}\right)+\frac{x_{T}\left(\bar{Y}_{l}\right)-x_{L}\left(\bar{Y}_{l}\right)}{2}[1-\cos (k \pi / m)]
$$

with a similar expression for $\bar{X}_{k}$. Note that $X_{k}, \bar{X}_{k}$ now also vary with the span-wise index $l$, since the local chord is assumed to be that corresponding to the (Chebyshev) midpoint $\bar{Y}_{l}$ at the present span-wise position. For simplicity, we assume here that the same number $m$ of chord-wise panels is used at each such position, although there is no reason in principle why $m$ could not be also allowed to vary with $l$, and that might lead to a more economical distribution of panels in some cases.

The end panels $k=1, m$ clearly do not fit closely to curved edges. For example, the left edge at $k=1$ is at $x=X_{0}=x_{L}\left(\bar{Y}_{l}\right)$, the (Chebyshev) mean position of a locally slanted leading edge. In general, this slanted edge cuts across the left side of the panel, the rectangular panel excluding a triangular piece that should be present, and including a triangular piece that should not. We hope that the aerodynamic effects of these inclusions and exclusions cancel out in the average for that panel, but cannot be sure that they will.

In view of the fact that we are using a Chebyshev grid where the edge 
rectangles are deliberately made smaller than others, it is also possible for the fit to slanted edges to be worse than this, in that a slanted edge could impact not just the end panels $k=1, m$, but also adjacent panels $k=2, m-1$, etc. To avoid this, we must panelise more finely in the span-wise direction than the chordwise direction, i.e., in principle have $n \gg m$. Otherwise, solution for arbitrary convex planforms proceeds essentially as if the planform was rectangular.

Typical results for the lift coefficient slope of a "circular" wing are 1.793536 from $(m, n)=(10,20)$ and 1.792474 from $(m, n)=(10,30)$. The computations suggest that the convergence with respect to $n$ is like $n^{-1}$, so that the extrapolated estimate for $(m, n)=(10, \infty)$ from the above results is 1.790350 . This is better than 4-figure agreement with the exact value 1.790750 of Hauptmann and Miloh [5], but this agreement is again perhaps a fluke, and less satisfactory extrapolation occurs at other $n$ values. The nearly 3-figure accuracy obtained by the actual computation for the $(10,20)$ panelisation of Figure 8 is perhaps representative of what is achievable by this naive procedure, and more work is needed if this method is to be competitive for general non-rectangular planforms.

\section{Acknowledgements}

This work was supported by the Australian Research Council. I acknowledge useful discussions with Leo Lazauskas.

\section{References}

[1] H. Ashley and L. Landahl, Aerodynamics of wings and bodies (Addison-Wesley, New York, 1965).

[2] M. Van Dyke, Perturbation methods in fluid mechanics (Academic Press, New York, 1964, Annotated edition, Parabolic Press, Stanford, 1975).

[3] N. T. Frink, "Lifting-surface theory for skewed and swept subsonic wings", J. Aircraft 19 (1982) 519-524.

[4] Jean-Luc Guermond, "A generalized lifting-line theory for curved and swept wings", $J$. Fluid Mech. 211 (1990) 291-317.

[5] A. Hauptmann and T. Miloh, "On the exact solution of the linearized lifting-surface problem of an elliptic wing", Quart. J. Mech. Appl. Math. 39 (1986) 41-66.

[6] J. E. Kerwin and C.-S. Lee, "Prediction of steady and unsteady marine propellor performance by numerical lifting surface theory", Soc. Nav. Arch. Mar. Engrs Trans. 86 (1978) 218-253. 
[7] B. Koemiawan, "Numerical solution of Prandtl's lifting-line equation", M. Sc. Thesis, University of Adelaide, 1992.

[8] R. P. Oertel, "The steady motion of a flat ship, including an investigation of local flow near the bow", Ph. D. Thesis, University of Adelaide, 1975.

[9] K. Stewartson, "A note on lifting line theory", Quart. J. Mech. Appl. Math. 13 (1960) 49-56.

[10] B. Thwaites, Incompressible aerodynamics (Oxford Univ. Press, Oxford, 1960).

[11] E. O. Tuck, "Application and numerical solution of Cauchy-singular integral equations", in The application and numerical solution of integral equations (eds. R. S. Anderssen et al.), (Sijthoff and Noordhoff, The Netherlands, 1980).

[12] E. O. Tuck, "Planing surfaces", in Numerical solution of integral equations (ed. M. Golberg), (Plenum Press, New York, 1990).

[13] E. Varley and J. D. A. Walker, "A method for solving singular integrodifferential equations", Inst. Math. Appl. J. Applied Math. 43 (1989) 11-45.

[14] H. T. Wang, "Comprehensive evaluation of six thin-wing lifting-surface computer programs", David Taylor Naval Research Center Rep. 4333 (1974).

[15] Hongbo Xü, "Potential flow solution for a yawed surface-piercing plate", J. Fluid Mech. 226 (1991) 291-317. 